\title{
An atypical presentation of cutaneous leishmaniasis
}

\author{
(D) Ahu Yorulmaz ${ }^{1}$, (D) Basak Yalcin ${ }^{1}$, (1) Ayse Nur Yilmaz ${ }^{2}$ \\ ${ }^{1}$ Ankara City Hospital, Department of Dermatology, Ankara, Turkey \\ ${ }^{2}$ Ankara City Hospital, Department of Pathology, Ankara, Turkey
}

Cite this article as: Yorulmaz A, Yalçın B, Yllmaz AN. An atypical presentation of cutaneous leishmaniasis. Anatolian Curr Med J 2021; 3(1); 66-68.

\begin{abstract}
Leishmaniasis is among the neglected tropical diseases, which are known to dominate in poor, rural populations living in tropical and subtropical climates. Recent data have demonstrated that neglected tropical diseases affect more than 1 billion people worldwide. Since we are facing one of the great refugee crises of all time, neglected tropical diseases are currently should be considered as a main public health problem at the global level. Cutaneous leishmaniasis may be confused with many other dermatoses since it is one of 'the great masquerader diseases'. As dermatologist we are among the first to challenge with perplexing cases of cutaneous leishmaniasis, we should be aware of the full range of clinical presentations of cutaneous leishmaniasis. Here, we have presented a case of atypical cutaneous leishmaniasis in a 32-year-old man.
\end{abstract}

Keywords: Leishmaniasis, atypical presentation, sporotrichoid

\section{INTRODUCTION}

Leishmaniasis is a vector-borne protozoan disease caused by flagellated protozoans of the genus Leishmania. Leishmaniasis is a worldwide disease prevalent in tropical and subtropical regions and endemic in more than 90 countries where 1.5 to 2 million new cases occur annually $(1,2)$. Leishmaniasis has been known as the great imitator' because of difficulty in diagnosis and as it can look like any other dermatoses (3). Here, we have presented a case of atypical cutaneous leishmaniasis, whose presentation was striking with linearly distributed multiple nodules, which was completely different from typical cutaneous leishmaniasis lesions.

\section{CASE REPORT}

A 32-year-old Syrian man visited our outpatient clinic with a 2-month history of erythematous lesions on his left arm. He was otherwise healthy with no other significant symptoms and past medical history. A dermatological examination demonstrated linearly distributed three erythematous infiltrated nodules on his left proximal upper extremity (Figure 1).

The middle lesion was greater in diameter with two identical peripheral ones. Laboratory investigations including complete blood and differential counts, erythrocyte sedimentation rate and full blood chemistry were within normal ranges. Histology of a lesional skin biopsy revealed dermal infiltrate of lymphocytes and granulomas composed of epithelioid histiocytes (Figure 2).

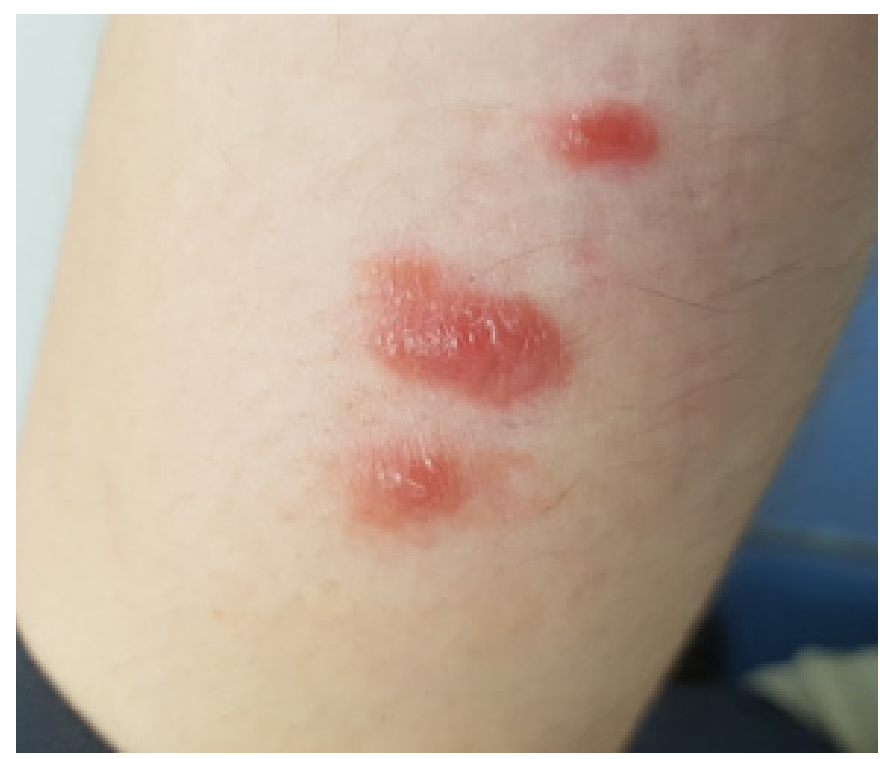

Figure 1. Bright red ertyhematous nodules without clinically evident epidermal changes 


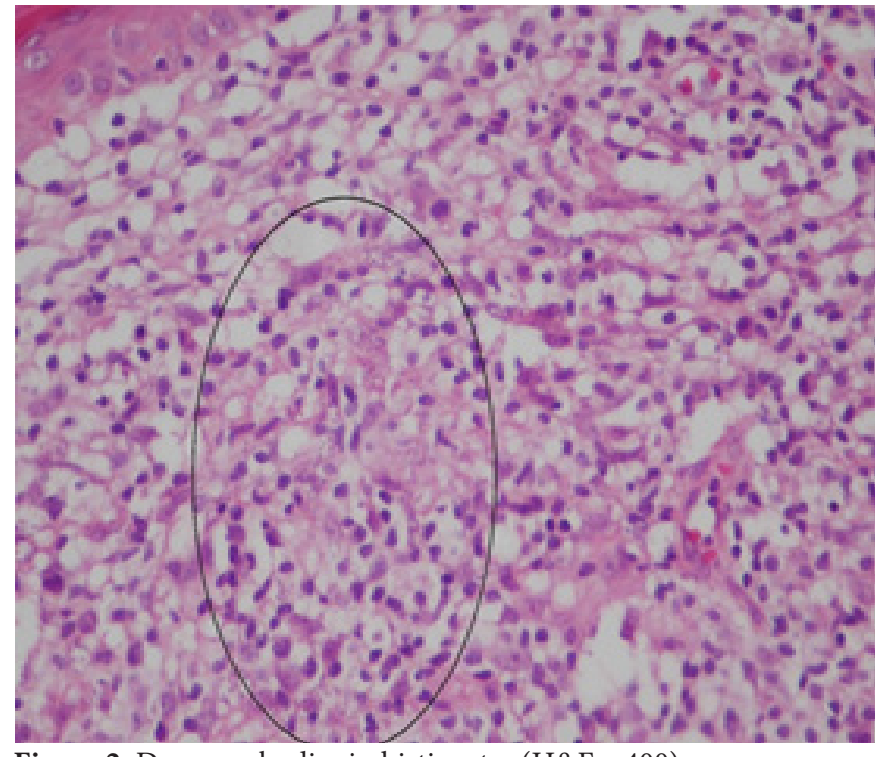

Figure 2. Donovan bodies in histiocytes (H\&E x 400)

Donovan bodies in histiocytes were detected in hematoxylin-eosin stained sections. Giemsa stain and CD1a immunohistochemistry confirmed the presence of intracytoplasmic Leishmania amastigotes (Figure 3, 4). Thus, based on the clinical and histopathological findings, a diagnosis of sporotrichoid cutaneous leishmaniasis was established and intralesional pentavalent antimonial injection was planned as a treatment protocol for the patient.

\section{DISCUSSION}

Cutaneous leishmaniasis is well-known to have a wide range of diverse clinical presentations. Up to now, the clinical classification of cutaneous leishmaniasis has been made depending on the pattern, behavior, and extent of the disease, which includes localized, mucocutaneous, diffuse, post kala-azar dermal, viscerotropic leishmaniasis and leishmaniasis recidivans. On the other hand, a different classification has been proposed for atypical lesions according to morphology, site, and number of lesions. Thus, a myriad of distinct terminologies has been evolved in the medical nomenclature of leishmaniasis. Zosteriform, rhynophymatous, chancriform, paronychial, sporotrichoid, erysipeloid, verrucous, acneiform, lupoid, eczematous and psoriasiform are some of the terminologies to describe atypical presentations of cutaneous leishmaniasis (4-6).

Sporotrichoid leishmaniasis is one of the atypical presentations of cutaneous leishmaniasis, which is characterized by primary lesion accompanied with lymphangitis and secondary satellite lesions around the main one. Sporotrichoid leishmaniasis has been rarely described in the literature. Since the clinical presentation is different from characteristic ulcerative papulonodules

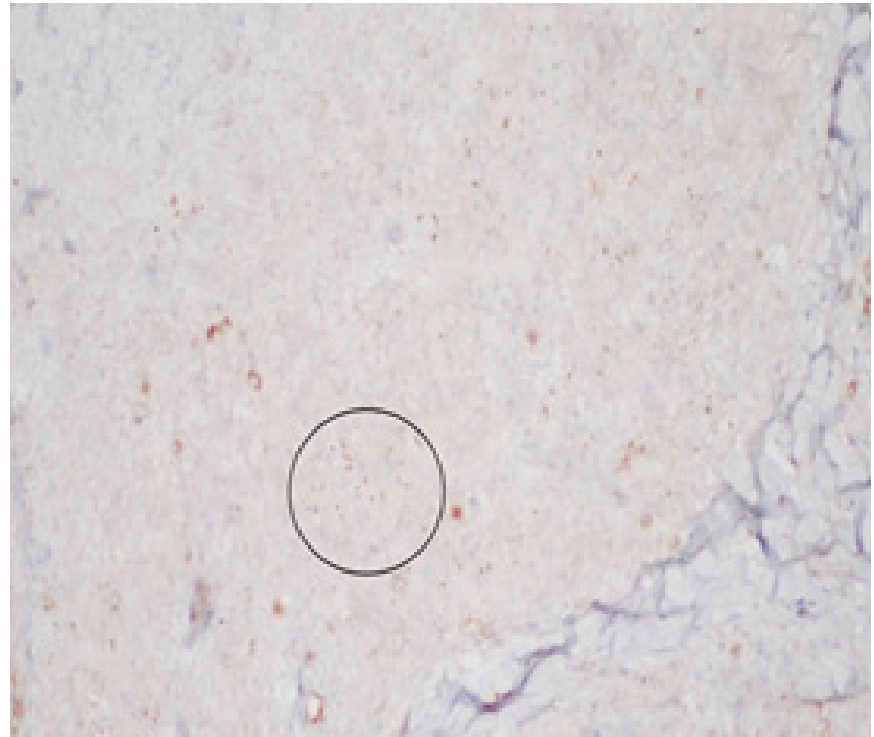

Figure 3. CDla positive amastigotes (X400)

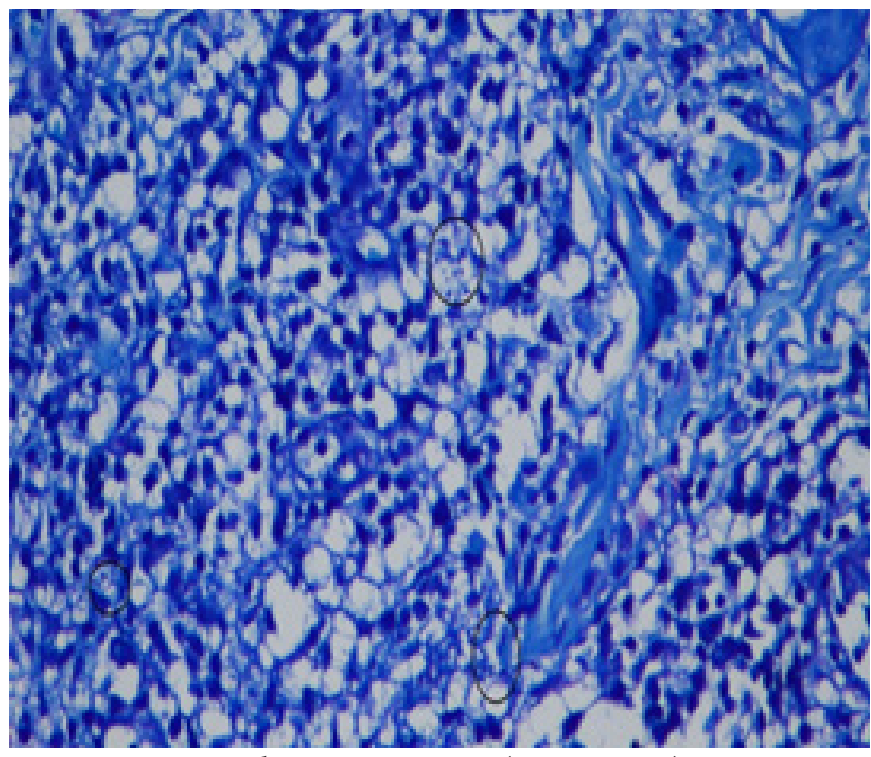

Figure 4. Intracytoplasmic amastigotes (GiemsaX400)

with raised border, clinicians generally remain suspicious about the diagnosis and miss it. However, with a detailed history of living or traveling to endemic areas, an indicative clue emerges and the quest for histopathological examination continues. Histopathological examination of lesions reveals typical noncaseating granulomas in the dermis and intracytoplasmic amastigote protozoans (713).

It is not clear why there are so many atypical presentations of cutaneous leishmaniasis. It has been suggested that host immune response and the pathogenic species of the protozoan are the main factors determining the disease outcome (6). Likewise, some authors have argued about the causative factors in the sporotrichoid dissemination of leishmaniasis. Traumatic insult, including therapies such as cryotherapy or intralesional pentavalent antimonial injections, has been questioned as a triggering factor for 
sporotrichoid spread. However, in some of the reports this relationship was not proved, rather sporotrichoid pattern had been observed without preceding therapies (13). Impaired cell-mediated immunity to leishmanial antigens has been investigated as an underlying cause in the dissemination of cutaneous leishmaniasis. Different levels of macrophage chemoattractant protein and macrophage inflammatory protein have been demonstrated in localized and diffuse leishmaniasis (14). These findings have implications for a possible causal immunological association between host and the parasite in the etiology of dissemination in leishmaniasis $(6,13,14)$.

Sporotrichoid leishmaniasis is within the differential diagnosis of leishmaniasis with multiple lesions. It is well-known that leishmaniasis typically presents with a solitary lesion, however, multiple lesions that resulted from multiple bites may also ocur (5). But in an incidence of multiple bites, lesions should be exclusively identical both in morphology and size. As it is seen in our patient, in sporotrichoid leishmaniasis there is a striking linear distribution with the primary lesion at the center and analog satellite lesions at the periphery. Here, we have described a patient with sporotrichoid leishmaniasis and with this particular presentation, we emphasize the importance of detailed dermatological examination in obscure clinical presentations.

\section{ETHICAL DECLARATIONS}

Informed Consent: Written informed consent was obtained from all participants who participated in this study.

Referee Evaluation Process: Externally peer-reviewed.

Conflict of Interest Statement: The authors have no conflicts of interest to declare.

Financial Disclosure: The authors declared that this study has received no financial support.

Author Contributions: All of the authors declare that they have all participated in the design, execution, and analysis of the paper, and that they have approved the final version.

\section{REFERENCES}

1. Steverding D. The history of leishmaniasis. Parasit Vectors 2017; 10: 82 .

2. Torres-Guerrero E, Quintanilla-Cedillo MR, Ruiz-Esmenjaud J, Arenas R. Leishmaniasis: a review. F1000Res 2017; 6: 750.

3. Bilgic Temel A, Murrell DF, Uzun S. Cutaneous leishmaniasis: a neglected disfiguring disease for women. Int J Womens Dermatol 2019; 5: 158-65.

4. Bari AU, Rahman SB. Many faces of cutaneous leishmaniasis. Indian J Dermatol Venereol Leprol 2008; 74: 23-7.
5. Bari AU. Clinical spectrum of cutaneous leishmaniasis: an overview from Pakistan. Dermatol Online J 2012; 18 : 4.

6. Carvalho LMV, Pimentel MIF, Conceição-Silva F, et al. Sporotrichoid leishmaniasis: a cross-sectional clinical, epidemiological and laboratory study in Rio de Janeiro State, Brazil. Rev Inst Med Trop Sao Paulo 2017; 59: e33.

7. Ntasou AA, Grummich H, Fischer K, et al. Mucosal relapse one year after complete remission of cutaneous sporotrichoid leishmaniasis due to Leishmania braziliensis. Eur J Dermatol 2016; 26: 94-5.

8. Walsh DS, Balagon MV, Abalos RM, et al. Multiple lesions of sporotrichoid leishmaniasis in a Filipino expatriate. J Am Acad Dermatol 1997; 36: 847-9.

9. Kibbi AG, Karam PG, Kurban AK. Sporotrichoid leishmaniasis in patients from Saudi Arabia: clinical and histologic features. JAAD 1987; 17: 759-64.

10. Erdogan FG, Cakır AG, Gokoz O, Gurler A. A case of sporotrichoid cutaneous leishmaniasis. Turkderm 2011; 45: 1003.

11. Bhardwaj K, Ghate S, Dandale A, Dhurat R. Sporotrichoid papulo-nodules with retiform rash:unusual presentation of leishmaniasis. Int J Infect Dis 2016; 45: 354.

12. Ayatollahi J. sporotrichoid cutaneous leishmaniasis in Central Iran. Iranian J Med Sci 2006; 31: 173-6.

13. Cozzani E, Satta R, Fausti V, Cottoni F, Parodi A. Cutaneous sporotrichoid leishmaniasis resistant to pentavalent antimonial therapy: complete resolution with itraconazole. Clin Exp Dermatol 2011; 36: 49-51.

14. Al-Qurashi AR, Ghandour AM, Osman M, Al-Juma M. Dissemination in cutaneous leishmaniasis due to leishmania major in different ethnic groups in Saudi Arabia. Int J Dermatol 2000; 39: 832-6. 